\title{
INCIDÊNCIA DE EMPIEMA PLEURAL NO TRAUMA ISOLADO DO TÓRAX COM E SEM USO DA ANTIBIOTICOTERAPIA
}

\author{
INCIDENCE OF EMPYEMA IN ISOLATED CHEST TRAUMA WITH AND \\ WITHOUT ANTIBIOTIC THERAPY
}

\author{
Mauro José Fontelles ${ }^{1}$ \\ Mario Mantovani, TCBC-SP ${ }^{2}$
}

\begin{abstract}
RESUMO: Objetivo: O objetivo do presente estudo foi avaliar a incidência de empiema pós-drenagem pleural fechada, nos pacientes com lesão isolada do tórax, com e sem uso da antibioticoterapia associada. Método: Utilizando o modelo estatístico de acompanhamento de coortes, os autores analisaram 167 pacientes acometidos por lesão traumática do tórax. Dois grupos foram selecionados para o estudo. O grupo controle incluiu 104 $(62,3 \%)$ pacientes sem uso da antibioticoterapia e, no grupo experimental, $63(37,7 \%)$ pacientes receberam a cefalotina sódica no pós-operatório (500mg IV - 6/6h). Resultados: Entre os pacientes estudados, 12 (7,2\%) apresentavam trauma fechado; $98(58,7 \%)$, ferimento por arma branca; $41(24,6 \%)$ ferida por projétil de arma de fogo e $16(9,5 \%)$ lesões por outros agentes vulnerantes. Entre os pacientes do grupo controle o tempo médio de

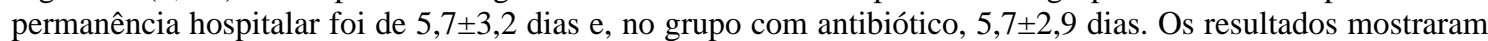
que oito $(4,7 \%)$ pacientes evoluíram com quadro de empiema pleural, sendo sete $(6,7 \%)$ casos no grupo controle e apenas um $(1,5 \%)$ no grupo experimental $(\mathrm{p}=0,26)$. O hemotórax coagulado foi a complicação não infecciosa mais freqüente, incidindo em 21 (12,5\%) pacientes. Conclusão: No presente estudo, os resultados mostram que o uso da antibioticoterapia não se mostrou eficaz em diminuir a incidência de empiema pleural nos pacientes submetidos à drenagem pleural pós-traumática.
\end{abstract}

Descritores: Empiema pleural; Trauma torácico; Drenagem pleural.

\section{INTRODUÇÃO}

O empiema pleural ou piotórax é definido como um processo infeccioso que acomete o espaço pleural com acúmulo de material purulento ${ }^{1,2}$. Sua incidência, nos pacientes vitimados por trauma, varia, segundo dados da literatura especializada, de um a $16 \% \%^{3-5}$, com grande parte dos autores referindo um valor abaixo de $3 \%{ }^{6-9}$. Esta tem sido uma condição com sérias repercussões em virtude da morbidade e mortalidade elevadas, muitas vezes ocorrendo em razão de procedimentos como toracocentese ou drenagem pleural mal conduzidas, além de outros fatores que influenciam no aparecimento desta complicação ${ }^{1,7-11}$.
Assim, o objetivo deste estudo foi analisar a incidência de empiema pleural nos pacientes portadores de lesão traumática limitada à região do tórax e submetidos, unicamente, à drenagem pleural fechada, com e sem uso da antibioticoterapia.

\section{MÉTODO}

Analisou-se, prospectivamente, um total de 167 pacientes acometidos por trauma isolado do tórax e submetidos à drenagem pleural fechada, sendo classificados em dois grupos selecionados através de amostragem randômica' ${ }^{12}$, para um estudo de acompanhamento de

1. Mestre em Cirurgia do Trauma e Aluno do Curso de Doutorado do Departamento de Cirurgia da Faculdade de Ciências Médicas da UNICAMP.

2. Professor Titular e Chefe da Disciplina de Cirurgia do Trauma do Departamento de Cirurgia da Faculdade de Ciências Médicas da UNICAMP.

Recebido em 31/7/2000

Aceito para publicação em 6/3/2001

Trabalho realizado pela Disciplina de Cirurgia do Trauma do Departamento de Cirurgia da Faculdade de Ciências Médicas da UNICAMP - Campinas - SP. 
coortes. Foram considerados pertencentes ao grupo controle, 104 pacientes aos quais não foi administrada a antibioticoterapia e, como grupo experimental, 63 pacientes fazendo uso, no pós-operatório imediato, da cefalotina sódica, administrada na dose de $500 \mathrm{mg}$, intravenosa, a cada seis horas, iniciada imediatamente antes da drenagem e continuada até 12 horas após a retirada do dreno ou, no máximo, por cinco dias.

A drenagem torácica foi sempre do tipo fechada, sob selo d'água, com utilização de dreno tubular multiperfurado 38/40 French, conforme preconizado pelo SAVT - Suporte Avançado de Vida no Trauma (ATLS - Advanced Trauma Life Support) ${ }^{13}$.

O diagnóstico do empiema foi confirmado, tomando-se como base os achados clínicos de febre (temperatura axilar $>38^{\circ} \mathrm{C}$ ), sinais de toxemia do paciente, derrame pleural persistente com aspecto turvo e purulento do líquido coletado. Os achados radiológicos de líquido no espaço pleural e identificação de microrganismos na secreção, caracterizaram o quadro.

Foram excluídos do estudo os pacientes com lesões associadas, localizadas em outras regiões anatômicas e aqueles submetidos à toracotomia; pacientes menores de 13 anos; portadores de doenças associadas; mulheres grávidas e pacientes transferidos durante o tratamento.

As análises de correlação foram realizadas pela aplicação do teste de Qui-quadrado em tabelas de contingência de dupla entrada. Tratando-se com freqüência menores que cinco ou com pequenas amostragens, este teste foi substituído pelo teste de probabilidade exata de Fisher, a fim de se evitarem distorções na apreciação da significância estatística. Em todas as análises empregou-se o nível alfa de $5 \%(\mathrm{p} \leq 0,05)$.

\section{RESULTADOS}

A idade observada nos pacientes do grupo controle variou dos 13 aos 53 anos, com média de 26,8 $\pm 8,9$ anos, sendo constituído por $97(93,3 \%)$ homens e sete $(6,7 \%)$ mulheres. No grupo experimental a faixa etária ficou com-

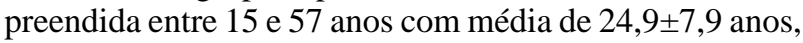
representado por $62(98,4 \%)$ pacientes do sexo masculino e apenas um $(1,6 \%)$ do sexo feminino $(1,6 \%)$. No total, a faixa etária mais acometida variou dos 13 aos 29 anos, correspondendo a $71,9 \%$ dos pacientes estudados.

Analisando-se a freqüência dos tipos de trauma, verificou-se que $155(92,8 \%)$ apresentaram lesão penetrante e apenas $12(7,2 \%)$ pacientes eram portadores de trauma fechado. Nos dois grupos estudados, $83(49,7 \%)$ pacientes apresentavam diagnóstico de hemotórax, em $16(9,6 \%)$ encontrou-se pneumotórax e $68(40,7 \%)$ eram portadores de hemopneumotórax (Tabela 1).

Com base nos achados cirúrgicos, todos os doentes tiveram os índices de trauma calculados. O valor médio do RTS (Revised Trauma Score), à admissão, foi avaliado em $7,4 \pm 0,3(5,4-7,6)$, o ISS (Injury Severity Score) com $10,1 \pm 2,5(09-16)$ e o TRISS 98,9 (97\% - 99\%).

O tempo de permanência hospitalar variou de três a

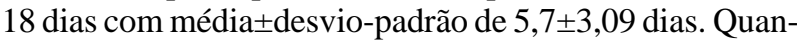
do avaliado o tempo de internação apenas para os pacientes que evoluíram com quadro de empiema pleural, este valor aumenta para 14,0 $\pm 1,8$ dias.

O empiema pleural esteve presente em oito $(4,7 \%)$ dos pacientes avaliados, sendo sete $(6,7 \%)$ casos no grupo controle e apenas um $(1,5 \%)$ caso no grupo experimental, relação esta sem significância estatística (Tabela 2).

Entre as complicações decorrentes de falha no sistema de drenagem, com evacuação incompleta da cavidade pleural, o hemotórax coagulado foi a mais freqüente, inci-

Tabela 1

Número de casos conforme as indicações para toracostomia de acordo com o diagnóstico e o grupo de pacientes estudados

\begin{tabular}{|c|c|c|c|c|}
\hline \multirow[b]{2}{*}{ Diagnóstico } & \multicolumn{2}{|c|}{ Controle } & \multicolumn{2}{|c|}{ Experimental } \\
\hline & $N^{o}$ & $\%$ & $N^{o}$ & $\%$ \\
\hline Hemotórax (total) & 55 & 52,9 & 28 & 44,4 \\
\hline Arma branca & 30 & 28,9 & 14 & 22,4 \\
\hline Arma de fogo & 21 & 20,2 & 10 & 15,8 \\
\hline Contusão & 02 & 1,9 & 03 & 4,7 \\
\hline Outros & 02 & 1,9 & 01 & 1,5 \\
\hline Pneumotórax (total) & 13 & 12,5 & 03 & 4,7 \\
\hline Arma branca & 08 & 7,7 & 02 & 3,2 \\
\hline Arma de fogo & - & - & - & - \\
\hline Contusão & 03 & 2,9 & - & - \\
\hline Outros & 02 & 1,9 & 01 & 1,5 \\
\hline Hemopneumotórax (total) & 36 & 34,6 & 32 & 50,9 \\
\hline Arma branca & 26 & 25,0 & 18 & 28,8 \\
\hline Arma de fogo & 03 & 2,9 & 07 & 11,1 \\
\hline Contusão & 02 & 1,9 & 02 & 31 \\
\hline Outros & 05 & 4,8 & 05 & 7,9 \\
\hline
\end{tabular}


Tabela 2

Número de casos conforme a incidência de empiema pleural de acordo com o grupo de pacientes estudados

\begin{tabular}{|c|c|c|c|c|c|c|}
\hline \multirow[b]{2}{*}{ Complicação } & \multirow[t]{2}{*}{ Grupo } & \multicolumn{2}{|c|}{ Controle } & \multicolumn{2}{|c|}{ Experimental } & \multirow[b]{2}{*}{ Total } \\
\hline & & № & $\%$ & $N^{o}$ & $\%$ & \\
\hline Com empiema & & 07 & 6,7 & 01 & 12,5 & 08 \\
\hline Sem empiema & & 97 & 93,3 & 62 & 39,0 & 159 \\
\hline Total & & 104 & & 63 & & 167 \\
\hline
\end{tabular}

$P=0,26$

OR $=4,47$

IC95\% $(0,53-99,10)$

dindo em 21 (12,5\%) pacientes. Destes, cinco (2,9\%) apresentaram empiema pleural no pós-operatório $(\mathrm{p}=0,002)$.

No total de oito pacientes com empiema pleural, a cultura do líquido drenado mostrou-se negativa em dois $(25 \%)$ casos e a bactéria mais freqüente foi a Klebsiela pneumoniae, com três casos, seguida pelo Staphylococcus aureus e Serratia marcescens com dois e um casos, respectivamente.

\section{DISCUSSÃo}

A contaminação da cavidade pleural pode ocorrer por disseminação hematogênica, linfática ou pela inoculação direta de bactérias ${ }^{1}$. Nos traumas torácicos, o empiema desenvolve-se pela inoculação direta através de uma ferida penetrante, pela persistência de corpos estranhos contaminados no interior da cavidade pleural, pela presença de fístula broncopleural (a árvore brônquica é sempre contaminada) ou pela contigüidade com uma broncopneumonia que ocorra durante a evolução do paciente, o que é bastante comum ${ }^{14}$. Neste sentido, com intuito de evitar esta complicação, é imprescindível a evacuação completa e asséptica do conteúdo hemático da cavidade que, além de constituir-se em excelente meio de cultura, restringe a expansão pulmonar com prejuízo da função respiratória ${ }^{8,9,15}$.

Um outro aspecto, relativo à incidência de empiema pleural pós-traumático, diz respeito ao uso de antibióticos associados à drenagem torácica, fato este ainda bastante controverso, segundo dados da literatura ${ }^{1,3,4,6,9,16}$.

LeBlanc et al. ${ }^{6}$, com uso da cefapirina sódica, registraram 2,2\% de empiema nos pacientes sem o uso do antibiótico. LoCurto et al. ${ }^{17}$, em estudo semelhante, encontraram $13,3 \%$ desta complicação no grupo daqueles que não fizeram uso da cefoxitina no pós-operatório, ao passo que, no grupo com uso da droga, não houve ocorrência da infecção. Sriussadaporn et al. ${ }^{18}$, num estudo de 42 pacientes com trauma torácico fechado, dos quais 18 receberam antibiótico, encontraram três casos de empiema pleural, com um caso $(5,5 \%)$ no grupo que recebeu antibiótico e dois casos $(8,3 \%)$ nos demais pacientes. Estes autores concluíram não haver diferença estatística significativa na incidência de empiema entre os grupos estudados.
No presente trabalho, no grupo de 63 pacientes com uso da antibioticoterapia, apenas um $(1,5 \%)$ evoluiu com empiema pleural; no grupo de 104 pacientes sem uso da cefalotina, sete $(6,7 \%)$ evoluíram com esta complicação, não apresentando, portanto, significância estatística, quando comparados estes resultados $(\mathrm{p}=0,26)$.

Grover et al. ${ }^{19}$, afirmaram que a presença de microrganismo no material drenado com ausência de sinais clínicos não caracteriza um quadro de infecção, ao passo que a cultura pode apresentar-se negativa em pacientes com quadro de empiema francamente instalado, fato este em razão da infecção pleural por agentes anaeróbicos de difícil identificação, requerendo técnicas especiais para seu cultivo. Eddy et al. ${ }^{7}$, chamaram a atenção para o estudo bacteriológico da secreção, alertando para o fato de que alguns autores cultivam o líquido pleural, definindo como infecção o crescimento bacteriano sem caracterizar, entretanto, a invasão tecidual, tratando-se apenas de simples colonização. Mandal et al. ${ }^{20}$, também relataram pacientes com sinais clínicos e radiológicos de empiema, porém com culturas negativas, referindo uma incidência de $28 \%$ destes casos.

Nesta casuística, entre os oito pacientes que evoluíram com empiema pleural no pós-operatório, a cultura da secreção foi negativa em dois $(25 \%)$ casos, estando este valor compatível com a literatura consultada. A Klebsiela pneumoniae foi o agente bacteriano cultivado mais freqüente, aparecendo em três dos pacientes pesquisados.

Um fator adicional para a grande variação da flora bacteriana no empiema pleural está relacionado à seleção do caso estudado que, segundo alguns autores, depende do antibiótico em uso por tempo prolongado e do ambiente hospitalar, visto que esses fatores modificam tanto a flora normal quanto a patológica, resultando em culturas estéreis em $16 \%$ a $53 \%$ dos casos, ou mesmo levando à resistência bacteriana ${ }^{7,14}$. Neste sentido, o $S$. aureus tem tido especial interesse como agente usualmente encontrado nos empiemas que acometem pacientes jovens, ao passo que, uma flora mista é mais comum nos pacientes submetidos a procedimentos cirúrgicos como a toracotomia ou laparotomia por lesões do trato gastrointestinal ${ }^{20}$.

Richardson et al. ${ }^{2}$, referiram a falência ou demora na indicação da drenagem intercostal como o principal fator de risco envolvido no processo do desenvolvimento do empiema pleural, em razão da incompleta reexpansão pul- 
monar e do material coletado (hemotórax coagulado) que propicia um meio de cultura ideal para o desenvolvimento bacteriano. Neste sentido, a toracotomia ou mesmo a toracoscopia, quando disponíveis, estão indicadas. A utilização da toracoscopia, realizada precocemente, concomitante com o procedimento de drenagem, vem reduzindo de modo considerável este tipo de complicação e suas consequiências, em razão do diagnóstico mais acurado das possíveis lesões intracavitárias e da evacuação completa do espaço pleural ${ }^{2,21}$.

$\mathrm{Na}$ presente pesquisa, a evacuação incompleta da cavidade pleural com hemotórax coagulado foi o principal fator envolvido na evolução do empiema. Neste caso, o uso do antibiótico mostrou-se favorável ao impedir este tipo de evolução $(\mathrm{p}=0,002)$.

O tempo de permanência do dreno também é fator importante, pois aumenta de modo significativo a incidência de infecção após 72 horas de sua inserção na cavidade pleural. Em virtude deste fato, todos os esforços devem ser direcionados para a remoção do mesmo assim que tenha cumprido sua função. Eddy et al. ${ }^{7}$, relataram que $75 \%$ dos drenos, em pacientes que não desenvolveram empiema, foram retirados dentro de 24 a 72 horas, e $90 \%$ foram removidos dentro de 96 horas. Ao contrário, nos casos que apresentaram empiema, apenas $25 \%$ dos drenos foram retirados antes de 72 horas de drenagem. No estudo de LoCurto et al. ${ }^{17}$, nos pacientes aos quais foi administrada antibioticoterapia, o tempo de drenagem foi menor, quando comparado com o grupo sem antibiótico. Neste sentido, Gilsanz et al. ${ }^{22}$ comprovaram, através de estudos radiológicos, que, após 48 horas de drenagem, já se pode encontrar sinais de espessamento, com nítidos indícios de processo inflamatório do folheto pleural, em razão da irritação provocada pelo dreno.

Na presente casuística, o tempo de permanência do dreno na cavidade pleural mostrou-se fortemente associado com o aparecimento do empiema no pós-operatório, resultado este compatível com a literatura consultada $(\mathrm{p}<0,00001)$.

A técnica cirúrgica empregada, quando da inserção do dreno, é fundamental na profilaxia da infecção, devendo ser instituídos todos os cuidados de assepsia e anti-sepsia, evitando-se assim o carreamento de bactérias para o espaço pleural ${ }^{3,7}$. A contaminação pode ocorrer não só pelo próprio mecanismo do trauma mas também pela não observância dos princípios técnicos, quando da inserção do dreno. A presença de corpo estranho no interior da cavidade torácica é outro componente a ser observado que aumenta a possibilidade de infecção. Portanto, cuidado especial deve ser dispensado para identificação e remoção dos mesmos, se introduzidos no espaço pleural em conseqüência ao trauma, sob o risco de infecção, mesmo após drenagem e antibioticoterapia adequadas ${ }^{2,7,20}$.
Neste trabalho, a opção pela drenagem pleural, conforme a técnica preconizada pelo ATLS, considerou ser esta a mais apropriada para tratar pacientes com trauma torácico e, atualmente, é a mais empregada em razão da menor incidência de complicações associadas.

Avaliando o mecanismo da lesão, na presente pesquisa, observou-se que os pacientes que sofreram traumatismo fechado da região torácica evoluíram com maior incidência de empiema pleural (10\%) quando comparados com as lesões por arma de fogo $(2,5 \%)$ e arma branca $(6,5 \%)$, valores estes sem significância estatística.

A idade do paciente é também um fator de risco a ser considerado no desenvolvimento do empiema pleural póstraumático. Pacientes idosos, mais amiúde, apresentam diminuídas suas reservas fisiológicas cardiovascular e respiratória, freqüentemente com doenças crônicas associadas, estando, portanto, mais propensos a reter secreções pulmonares com desenvolvimento de atelectasias e pneumonias subseqüentes. Estes pacientes possuem maior grau de imunodepressão, com resposta deficiente ao trauma, quando comparados com a população mais jovem. Ainda que as vítimas de lesões torácicas, em sua maioria, pertençam à faixa etária mais jovem, portanto com menor incidência de patologias associadas, o trauma em si induz a graus diversos de imunodepressão que estão na dependência da gravidade do mesmo, colocando estes pacientes, igualmente, no grupo de risco para o desenvolvimento de complicações infecciosas ${ }^{17,18,23}$.

$\mathrm{Na}$ presente casuística, 120 (71,9\%) dos pacientes avaliados pertenciam à faixa etária mais jovem da população, com idade compreendida entre 13 e 29 anos, portanto compatível com os dados relatados na literatura.

Outros fatores associados ao trauma, do mesmo modo, podem contribuir para depressão do sistema imunológico. Procedimentos cirúrgicos, técnicas invasivas de diagnóstico ou terapêuticas, uso prévio de medicamentos (antibióticos, corticóides e outros) ou drogas, como álcool e fumo, devem ser pesquisados. O risco subseqüente de infecção também aumenta na presença de lesões associadas com hemorragia e choque de duração prolongada na admissão e deterioração do nível de consciência por trauma cranioencefálico. Desta forma, afirmam diversos autores, a prevenção da infecção do espaço pleural é melhor determinada pela evacuação completa e retirada precoce do dreno torácico ${ }^{5,7,17,23}$

Portanto, analisando os resultados encontrados, podese concluir que a incidência de pacientes que evoluíram com empiema pleural, neste estudo, foi compatível com os valores relatados na literatura, estando presente em oito $(4,7 \%)$ dos 167 pacientes estudados, e o uso do antibiótico não mostrou benefício estatisticamente significante em evitar este tipo de complicação.

\section{ABSTRACT}

Background: The aim of this study was to evaluate the incidence of pleural empiema associated with tube thoracostomy on patients with isolated chest injury, with or without the use of associated antibiotic therapy. 
Method: Using cohorts accompaniment statistical model, the authors analysed 167 patients with blunt or penetrating chest trauma. Two groups were selected for this study. Control group included 104 (62.3\%) patients without antibiotic therapy; the experimental group, 63 (37.7\%) patients, received cefalotin postopertively (500mg IV; every 6 hours). Results: Twelve (7.2\%) presented blunt trauma; 98 (58,7\%) were stabbed, 41(24.6\%) were wounded by fire arms; 16 (9.5\%) injuries were associated with other type of accidents. The average length of stay

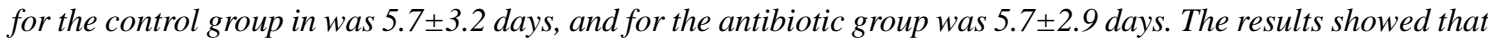
eight patients evolved with the pleural empyema, being seven cases from the control group, and only one from the experimental group $(p=0.02)$. Clotted hemotorax was the most frequent non infectious complication occurring on $21(12.5 \%)$ patients. Conclusions: The use of antibiotics were not effective on lowering the incidence of pleural empyema on patients that submitted to post-traumatic pleural drainage.

Key Words: Empyema; Thoracic trauma; Pleural drainage.

\section{REFERÊNCIAS}

1 Marsico GA - Empiema Pleural. JBM, 1994, 67: 209 - 18.

2 Richardson JD, Miller FB, Carrilo EH - Complex Thoracic Injuries. Surg Clin North Am, 1996, 76:725 - 46

3 Millikan JS, Moore EE, Steiner E, et al. - Complications of Tube Thoracostomy for Acute Trauma. Am J Surg, 1980, 140:738 - 41 .

4 Miller KS, Sahn AS: Chest Tubes - Indications, technique, management and complications. Chest, 1987, 91:258 -64 .

5 Etoch SW, Bar-Natan MF, Miller FB, et al. - Tube Thoracostomy: factors related to complications. Arch Surg, 1995, 130:521 - 26.

6 LeBlanc KA, Tucker WY - Prophylatic Antibiotic and Closed Tube Thoracostomy. Surg Gynecol Obstet, 1985, 160:259- 63 .

7 Eddy AC, Luna GK, Copass M - Empyema Thoracis in Patients Undergoing Emergent Tube Thoracostomy for Thoracic Trauma. Am J Surg, 1989, 157:494 - 97.

8 Saad JR, R. - Complicações das drenagens torácicas. In: Margarido NF, Saad JR R, Cecconello I, et al. - Complicações em cirurgia - Colégio Brasileiro de Cirurgiões. 1 ed. São Paulo: Robe Editorial, 1993, pp 325 -344 .

9 Botter M, Saad JR R, Giannini JA, et al. - Drenagem Pleural no Trauma Torácico. J Pneumol, 1996, 22:59 - 64.

10 Mavroudis C, Symmonds JB, Minagi H, et al. - Improved Survival in Management of Empiema Thoracis. J Thorac Cardiovasc Surg, 1981, 82:49 - 57.

11 DE LA Rocha AG - Empyema Thoracis. Surg Gynecol Obstet. 1982; 155:839-45.

12 Jekel JF, Elmore JG, Katz DL - Epidemiologia, Bioestatística e Medicina Preventiva. Porto Alegre: Artes Médicas Sul, 1999, 328 p.

13 AMERICAN COLLEGE OF SURGEONS - Manual de Suporte Avançado de Vida no Trauma - ATLS. 6. ed., rev. s. 1: Ministério da Saúde, 1997. 414 p.

14 Netto AC, Filomeno LT - Empiema Pleural. In: Raia AA, Zerbini E: Clínica Cirúrgica Alípio Corrêa Neto. 4. ed. rev. e ampl. São Paulo: Sarvier, 1988, pp 185 213.

15 Symbas PN - Chest Drainage Tubes. Surg Clin North Am, 1989, 69:41 - 46.

16 Fallon JR WF, Wears RL - Prophylatic Antibiotics For The Prevention Of Infectious Complications Including Empyema Following Tube Thoracostomy For Trauma: result of meta-analysis. J Trauma, 1992, 33:110 - 6.
17 LoCurto JJ, Tischler CD, Swan KG, et al. - Tube Thoracostomy and Trauma - Antibiotics or Not?. J Trauma, 1986, 12:1067 - 72 .

18 Sriussadaporn S, Poomsuwan P - Post-Traumatic Empiema Thoracis in Blunt Chest Trauma. J Med Assoc Thai, 1995, 78:393 - 398 .

19 Grover FL, Richardson JD, Fewel JG, et al. - Prophylatic Antibiotics in the Treatment of Penetrating Chest Wounds. J Thorac and Cardiovasc Surg, 1977, 4:528 -36 .

20 Mandal AK, Thadepalli H, Mandal AK, et al. - Posttraumatic Empiema Thoracis: A 24 - Year Experience at a Major Trauma Center. J Trauma, 1997, 5:764 - 71.

21 Normando JR R, Tavares MAF, Cunha AC, et al., O papel da Decorticação Pulmonar no Tratamento do Hemotórax Coagulado. Rev Paraense Med, 1995, 2:10 - 4.

22 Gilsanz V, Cleveland RH -: Pleural Reaction to Thoracostomy Tube. Chest, 1978, 74:167 - 9.

23 McRitchie DI, Matthews JG, Fink MP - Pneumonia in Patients With Multiple Trauma. Clin Chest Med, 1995, $1: 135-46$.

Endereço para Correspondência

Dr. Mario Mantovani

Rua José de Campos Salles, 650

13095-300 - Campinas - SP 